\title{
On a One-Particle in the Continuum Approach for the Photoreaction Cross-Sections.
}

\author{
S. S. Aimad
}

Istituto di Fisica dell'Università - Padova

Istituto Nazionale di Fisica Nucleare - Laboratori Nazionali di Legnaro (Padova)

(Nuovo Oimento A, 54, $129(1979)$ )

$\begin{array}{ll}\text { Present (incorrect) } & \text { Correct } \\ \text { continuous } & \text { continuum } \\ \text { ROBINSON } & \text { RoBson } \\ \text { ROBINson } & \text { RoBson } \\ \text { SURIUS } & \text { SARUIS } \\ v_{0}, v_{\text {s.o. }}, v_{\text {Coul }} & v_{\mathbf{0}}, v_{\mathrm{B}, 0 .}, v_{\text {Coul }} \\ H & H_{\mathbf{0}} \\ \text { tube } & \text { to be } \\ \nu, Q_{10} & v, \mid Q_{10} \\ \text { changed } & \text { channel }\end{array}$

